\title{
SINDROME DE BURNOUT EM PROFISSIONAIS DE ENFERMAGEM DE UM HOSPITAL DE URGÊNCIA/EMERGÊNCIA*
}

\author{
Sabrina de Souza PEREIRA ${ }^{1}$ \\ Priscilla Maria de Castro SILVA ${ }^{2}$ \\ Elisângela Braga de AZEVEDO ${ }^{3}$ \\ Elaine Braga FAUSTINO \\ Zuíla Mayara Nicolau de ARAÚJO \\ Maria de Oliveira FERREIRA FILHA ${ }^{6}$
}

${ }^{1}$ Enfermeira. Graduada pela Faculdade de Ciências Médicas de Campina Grande - PB.

2 Mestranda do Programa de pós-graduação em Enfermagem da Universidade Federal da Paraíba. Docente do Departamento de Enfermagem da Universidade Federal de Campina Grande - PB. João Pessoa (PB). Brasil. E-mail: priscillamcs@hotmail.com.

${ }_{3}^{3}$ Doutoranda em enfermagem pelo Programa de Pós-Graduação da Universidade Federal da Paraíba. Professora do Departamento de Enfermagem da Universidade Estadual da Paraíba e da Faculdade de Ciências Médicas. João Pessoa (PB). Brasil. E-mail: elisaaz@terra.com.br. Endereço: Pedro Soares da Silva, 55, Catolé. CEP: 58411-150. Campina Grande, Paraíba.

${ }^{4}$ Enfermeira da Estratégia Saúde da Família do Município de Campina Grande- PB. Especialista em Enfermagem do trabalho e em Urgência e Emergência. Email: elainebragab@bol.com.br

${ }^{5}$ Enfermeira. Graduada pela Universidade Estadual da Paraíba - UEPB. Email: zuila.mayara@hotmail.com

${ }^{6}$ Doutora em Enfermagem pela Universidade Federal do Ceará. Professora Adjunto IV da Universidade Federal da Paraíba. Líder do Grupo de Estudos e Pesquisas em Saúde Mental Comunitária da UFPB. João Pessoa (PB). Brasil. Email: marfilha@yahoo.com.br

Recebido em: 26/05/2014 - Aprovado em: 30/06/2014 - Disponibilizado em: 30/07/2014

\section{RESUMO}

Objetivou-se com este trabalho, avaliar o desenvolvimento da síndrome de burnout, fazendo um comparativo entre os profissionais do período diurno e noturno de um hospital de emergência de Campina Grande - PB. Trata-se de um estudo transversal e quantitativo. Foi aplicado em 40 profissionais de enfermagem o Maslach Burnout Inventory para diagnosticar a síndrome. Não houve diferenças significativas entre o desenvolvimento de exaustão emocional e despersonalização quando comparados ao turno de trabalho. Mais de $80 \%$ apresentaram sentimento de incompetência moderados. No local de trabalho os escores foram moderados para as três dimensões, tanto nos atuantes na emergência, quanto na UTI. Os pontos de desequilíbrio entre trabalhadores e o ambiente de trabalho confirmam desgaste profissional e propensão para a síndrome, uma vez que o nível moderado já serve de alerta para a observância da presença dos sinais e sintomas referentes à síndrome e permite que intervenções sejam realizadas, a fim de minimizar os danos do esgotamento e tencionar o compromisso com o trabalho, tornando-o mais eficiente e eficaz.

Descritores: Enfermagem. Esgotamento profissional. Serviço hospitalar de emergência.

\section{BURNOUT SYNDROME IN PROFESSIONAL NURSING AN EMERGENCY HOSPITAL / EMERGENCY}

\begin{abstract}
The objective of this study was to evaluate the development of burnout by making a comparison between daytime and nighttime nursing professionals in a hospital emergency Campina Grande - PB. This is a cross-sectional study and quantitative. Was applied in 40 nursing professionals the Maslach Burnout Inventory to diagnose. There were no significant differences between the development of emotional exhaustion and depersonalization when compared to the shift. Over $80 \%$ had moderate feeling of incompetence. In the workplace, scores were moderate for all three dimensions, both in working in emergency and in the UTI. The points of imbalance between workers and the working environment, professional wear and confirm propensity for the syndrome, since the moderate level serves as a warning to the observance of the presence of signs and symptoms related to the syndrome and allows interventions to be undertaken, the minimize the damage and the depletion will the commitment to making it work more efficiently and effectively.

Keywords: Nursing. Professional exhaustion. Hospital emergency Service.
\end{abstract}




\section{Introdução}

O trabalho é inquestionavelmente uma atividade milenar, que ao longo dos séculos vem sofrendo alterações na maneira como é realizado, graças ao advento da tecnologia desenvolvida, que a cada dia está sempre se inovando e se fazendo cada vez mais presente tanto no ambiente de trabalho, como no ambiente domiciliar.

A tecnologia não pode ser confundida com equipamentos ou aparelhos tecnológicos, já que para alguns autores, a tecnologia envolve saberes e habilidades, e os equipamentos se configuram como sendo a expressão da tecnologia, sendo estes resultantes dos saberes e habilidades, utilizados para a obtenção do produto final, ou seja, do aparelho tecnológico (ROCHA, et al, 2008).

Complementando a ideia supracitada, as tecnologias podem ser classificadas em leve, quando falamos de relações, acolhimento, gestão de serviços; em leve-dura, quando nos referimos aos saberes bem estruturados, como o processo de enfermagem; e dura, quando envolvem os equipamentos tecnológicos do tipo máquinas (ROCHA, et al, 2008).

O trabalho é sem dúvida fonte de prazer e realização para os que exercem esta atividade, mas, além disto, o trabalho em condições desfavoráveis pode levar ao desgaste físico, ao sofrimento psíquico e ao estresse (MASLACH; JACKSON, 1981). Quando o organismo é exposto rotineiramente a situações de estresse, ele torna-se mais propício ao desencadeamento de uma síndrome, denominada síndrome de Burnout.

O que muitos estudos revelam é um comum consenso no que diz respeito à definição da síndrome de Burnout, sendo esta uma síndrome de caráter psicossocial, desencadeada como mecanismo de defesa em resposta a situações crônicas de estresse, envolvendo pessoas em seu ambiente de trabalho (CARLOTTO; CÂMARA, 2008). A síndrome de Burnout acomete profissionais que atuam de forma direta com as pessoas e que estão expostos a um estresse crônico, permanecendo, na maioria das vezes, por um período de tempo bem prolongado (MASLACH; JACKSON, 1981).

Sendo assim, os profissionais de enfermagem são bastante susceptíveis ao desenvolvimento desta síndrome, uma vez que lidam diretamente com pessoas e geralmente fazem uso de uma carga emocional muito forte, pois constantemente estão expostos a situações de dor e sofrimento por parte dos usuários a quem prestam assistência, lidando também frequentemente com perdas. Além disso, os membros da equipe de enfermagem geralmente estão submetidos a cargas horárias excessivas, e em muitos casos também necessitam trabalhar em mais de uma unidade de serviço a fim de obter uma melhor qualidade de vida no que se refere à questão financeira (ROSA; CARLOTTO, 2005).

A ausência de um controle em relação aos profissionais que estejam desenvolvendo 
a síndrome de Burnout pode resultar em diminuição da produtividade, no aumento do absenteísmo, podendo até mesmo levar o profissional a abandonar a profissão, já que assim ele poderá evitar o contato direto com os pacientes (MULLER, 2004).

A síndrome de Burnout constitui-se em um dos grandes problemas psicossociais atuais, despertando interesse e preocupação não só por parte da comunidade cientifica internacional, mas também das entidades governamentais, empresarias e sindicais norte-americanas e européias, devido à severidade de suas consequências, tanto em nível individual como organizacional (MUROFUSE; ABRANCHES; NAPOLEÃO, 2005). Deste modo, o trabalho do enfermeiro, principalmente no âmbito hospitalar, está entre os que mais geram estresse em seus profissionais (LAUTERT, 1995). A classificação da Health Education Authority aponta que dentre todas as profissões do setor público, a enfermagem ocupa a quarta colocação no ranking das profissões mais estressante (STACCIARINI; TRÓCCOLL, 2001).

Nesse contexto, este estudo objetivou identificar, através de uma análise comparativa, a propensão de uma equipe de enfermagem diurna e noturna (emergência e U.T.I.), para a síndrome de Burnout, em um hospital de emergência e trauma de Campina Grande, PB, além de caracterizar o perfil sociodemográfico das equipes de enfermagem da emergência e da U.T.I., e verificar entre estas equipes (emergência e U.T.I.) a incidência dos sinais e sintomas referentes à síndrome de Burnout, fazendo um comparativo entre a incidência desses sintomas nos períodos diurno e noturno.

\section{Metodologia}

Trata-se de um estudo descritivo, transversal, quantitativo, realizado em um Hospital de Urgência e Emergência, localizado no município de Campina Grande, Paraíba, Brasil, que por questões éticas será referido neste artigo como Hospital X.

Utilizou-se para coleta de dados a técnica da observação direta extensiva. Foram utilizados dois questionários estruturados para a coleta dos dados. $\mathrm{O}$ primeiro destinou-se a caracterização do perfil sociodemográfico dos profissionais participantes da pesquisa. O segundo questionário utilizado foi o Maslach Burnout Inventory (MBI) na versão Human service survey. $\mathrm{O}$ referido instrumento foi desenvolvido por Maslach e Jackson traduzido e validado no Brasil por Lautert. Trata-se de um questionário autoaplicável, contendo 22 afirmações cujo objetivo é avaliar a ocorrência da síndrome nas suas três dimensões, exaustão emocional, despersonalização e sensação de realização reduzida com o trabalho, que foram respondidas através de uma escala do tipo Likert (BENEVIDES-PEREIRA， 2002). A aplicação dos instrumentos ocorreu em turnos e dias pré-estabelecidos, conforme a conveniência dos participantes da pesquisa e segundo a rotina de funcionamento do 
serviço, nos meses de agosto a setembro de 2009. O corpo de enfermagem da instituição era formado por 151 profissionais, dos quais, 51 compunha o serviço de emergência e UTI, onde foi realizada a pesquisa. Destes, 40 profissionais de enfermagem participaram do estudo, representando um percentual de $78,43 \%$ do total de servidores lotados nesses setores.

Os dados foram analisados com o auxílio estatístico, através do software SPSS versão 15.0, e os resultados foram apresentados em tabelas e de forma descritiva. Para classificação dos níveis altos de burnout optou-se pelo percentil 75, e para a dimensão reduzida da realização profissional foi aplicado o percentil 25 , já que essa dimensão se refere a uma escala reversa. Para identificação de associação entre a ocorrência do burnout e as variáveis sociodemográficas, profissionais e o ITRA foram aplicados o teste do qui-quadrado e o teste exato de Fisher, quando indicado. Foram considerados significativos os valores de $\mathrm{p}<0,05$.

Foram respeitados todos os preceitos da resolução 466/2012, que regulamenta as pesquisas envolvendo seres humanos. Este estudo foi aprovado pelo Comitê de Ética do Centro de Ensino Superior e Desenvolvimento/Faculdade de Ciências Médicas (CESED/FCM), em 14 de agosto de 2009, sob o no 1848.0.000.405-09.

\section{Resultados e Discussão}

Os dados esquematizados na tabela 1 revelam que houve uma predominância do sexo feminino (55\%) sobre o sexo masculino (45\%), há uma equivalência na distribuição por turnos de trabalho entre o diurno e o noturno, como pequeno percentual atuando em ambos os turnos. Um quantitativo de $50 \%$ dos profissionais entrevistados trabalhavam na instituição fonte da pesquisa durante o período diurno, enquanto $42,5 \%$ trabalhavam durante o período noturno e 7,5\% deles trabalhavam em ambos períodos.

A participação de respondentes oriundos da UTI superou o da emergência, com $52,5 \%$ dos respondentes pertencentes ao primeiro setor. No concernente ao acúmulo de vínculos de trabalho, tem-se que um considerável percentual atuava em pelo menos mais uma instituição de trabalho, o que também se verificou para a carga horária semanal de trabalho, com mais de $70 \%$ desempenhado suas atividades profissionais com carga horária semanal entre 20 e 40 horas. No tangente ao tempo de formação profissional e ao tempo de exercício profissional da instituição pesquisada, mais da metade dos respondentes atuava profissionalmente num período compreendido entre 1 e 10 anos. 
Tabela 1 - Distribuição dos dados coletados quanto ao perfil sócio-profissional dos profissionais de enfermagem participantes da pesquisa, Campina Grande, 2009.

\begin{tabular}{|c|c|c|}
\hline Variáveis sócio-profissionais & & $f(\%)$ \\
\hline \multirow{2}{*}{ Sexo } & Masculino & 45,0 \\
\hline & Feminino & 55,0 \\
\hline \multirow{3}{*}{ Turno de trabalho } & Diurno & 50,0 \\
\hline & Noturno & 42,5 \\
\hline & Diurno e noturno & 7,5 \\
\hline \multirow{2}{*}{ Local de trabalho } & Emergência & 47,5 \\
\hline & UTI & 52,5 \\
\hline \multirow{2}{*}{ Exerce atividade em outra instituição } & Sim & 67,5 \\
\hline & Não & 32,5 \\
\hline \multirow{4}{*}{$\begin{array}{l}\text { Carga horária semanal de trabalho na } \\
\text { instituição pesquisada }\end{array}$} & 20 a 40 & 70,0 \\
\hline & 41 a 60 & 15,5 \\
\hline & Mais de 60 & 7,5 \\
\hline & NR & 10,0 \\
\hline \multirow{5}{*}{ Tempo de formação profissional (em anos) } & Menos de 1 & 7,5 \\
\hline & 1 a 10 & 52,5 \\
\hline & 11 a 20 & 20,0 \\
\hline & Mais de 20 & 17,4 \\
\hline & NR & 2,5 \\
\hline \multirow{4}{*}{$\begin{array}{l}\text { Tempo de trabalho na instituição pesquisada } \\
\text { (em anos) }\end{array}$} & Menos de 1 & 15,0 \\
\hline & 1 a 10 & 60,0 \\
\hline & 11 a 20 & 20,0 \\
\hline & Mais de 20 & 5,0 \\
\hline
\end{tabular}

Fonte: Hospital X, Campina Grande-PB, 2009.

O exercício da enfermagem ao longo

da história sempre foi caracterizado pelo predomínio do gênero feminino, sendo ele considerado um trabalho tipicamente feminino durante todas as épocas (MULLER, 2004). A predominância das mulheres no cuidado com pacientes não é recente, pois desde séculos atrás era destinado a este gênero o cuidado aos enfermos. A partir daí a história da enfermagem pode ser construída tendo a mulher como pilar deste processo e o que se nota é que ao longo dos anos esta vem carregando adjetivos tais como: a bondade, a caridade, a paciência e a dedicação
Os resultados encontrados nessa pesquisa demonstram que o homem está penetrando nessa área de atuação e ocupando os cargos que antes eram ocupados majoritariamente por mulheres.

A correlação da subescala exaustão emocional com o turno de trabalho encontra-se descrita na tabela 2. Podemos observar que a média para os que trabalhavam no período diurno é de $17,7 \%$, para os que trabalhavam no período noturno a média é de $17,47 \%$ e os que trabalhavam em ambos os turnos, diurno e noturno, a média foi de $17,6 \%$.

(REISDORFER, 2002).

Tabela 2 - Subescala de exaustão emocional relacionada ao turno de trabalho

\begin{tabular}{llcc}
\hline $\begin{array}{l}\text { Turno de trabalho no } \\
\text { hospital }\end{array}$ & Escore & Frequência & $\%$ \\
\hline \multirow{2}{*}{ Diurno } & Baixo & 1 & 5 \\
& Moderado & 18 & 90 \\
& Alto & 1 & 5 \\
\hline & Total & 20 & 100 \\
\hline
\end{tabular}




\begin{tabular}{llcc}
\hline Noturno & Baixo & 1 & 6 \\
& Moderado & 15 & 88 \\
& Alto & 1 & 6 \\
\hline & Total & 17 & 100 \\
\hline Diurno e Noturno & & & 100 \\
\hline & Moderado & 3 & 100 \\
\hline
\end{tabular}

Fonte: Hospital X, Campina Grande-PB, 2009.

A tabela 3 por sua vez, apresenta os valores na sub-escala de despersonalização relacionados com o turno de trabalho dos profissionais. As médias encontradas revelam o valor de 7,95\% para o período diurno, o que o classifica em um nível moderado de despersonalização, de $9,47 \%$ para os profissionais do período noturno, o que também os classificam com tendo um nível moderado de despersonalização, e uma média de $6,66 \%$ para os profissionais que trabalhavam os dois turnos, os que os classificam como tendo um baixo grau de despersonalização.

Sendo a enfermagem uma profissão que necessita estar em pleno funcionamento durante 24 horas do dia, os sete dias da semana, em exercício contínuo e ininterrupto, o trabalho por turnos e noturno é então, de caráter obrigatório para os profissionais desta área, sobretudo os que atuam em ambientes hospitalares (PAFARO; MARTINO, 2004).

Tabela 3 - Sub-escala de despersonalização relacionada ao turno de trabalho

\begin{tabular}{llcc}
\hline $\begin{array}{l}\text { Turno de trabalho no } \\
\text { hospital }\end{array}$ & Escore & Frequência & \% \\
\hline \multirow{2}{*}{ Diurno } & & 10 & 50 \\
& Baixo & 7 & 35 \\
& Moderado & 3 & 15 \\
\hline & Alto & 20 & 100 \\
\hline \multirow{2}{*}{ Noturno } & Total & & 29 \\
& & 5 & 47 \\
\hline & Baixo & 8 & 24 \\
\hline & Moderado & 4 & 100 \\
\hline Diurno e Noturno & Alto & 17 & \\
& Total & & 67 \\
\hline & Baixo & 2 & 33 \\
\hline & Alto & 1 & 100 \\
\hline
\end{tabular}

Fonte: Hospital X, Campina Grande-PB, 2009.

A tabela 4 indica os escores da subescala de sentimento de incompetência profissional com relação ao turno de trabalho dos respondentes. A média diurna equivale a $17,85 \%$, a dos profissionais do período noturno é de $15,9 \%$, e para os que trabalhavam diurno e noturno a média corresponde a 17. Quanto maior o valor nessa sub-escala, menos as pessoas sentem-se ineficientes. Os valores medianos encontrados para os turnos de trabalhos citados permite enquadrá-los em um nível moderado de sentimento de incompetência profissional. 
Tabela 4 - Sub-escala de sentimento de incompetência profissional relacionada ao turno de trabalho

\begin{tabular}{llcc}
\hline $\begin{array}{l}\text { Turno de trabalho no } \\
\text { hospital }\end{array}$ & Escore & Frequência & $\%$ \\
\hline Diurno & Moderado & 17 & 85 \\
& Alto & 3 & 15 \\
\hline & Total & 20 & 100 \\
\hline & & 1 & 6 \\
\hline Noturno & Baixo & 16 & 94 \\
\hline & Moderado & 17 & 100 \\
\hline & Total & & 100 \\
\hline & & 3 & 100 \\
\hline
\end{tabular}

Fonte: Hospital X, Campina Grande-PB, 2009.

O setor que esses profissionais atuam também pode contribuir para o desenvolvimento da síndrome. A emergência e a U.T.I., de um hospital são setores críticos em que os profissionais lidam constantemente com o sofrimento humano e com frequentes perdas, uma vez que estes atendem pessoas em estado grave e com um grande comprometimento de suas funções vitais. Soma-se o elevado número de aparelhos com os quais os pacientes devem estar permanentemente ligados e para os quais os profissionais devem dedicar especial atenção, com a aquisição e manutenção de conhecimentos mínimos para o manuseio satisfatório dos mesmos. Além, frise-se, das demandas físicas existentes durante o tempo de trabalho, e a convivência com ritmos intensos de trabalho tanto nas UTI's, como nos serviços de emergência (BARROS, et al, 2008).

A tabela 5 exibe o resultado da subescala de exaustão emocional em relação ao local de trabalho dos respondentes da pesquisa. A média extraída revela uma pequena diferença nos valores, sendo a média da Emergência igual a 17,52\% e a média da U.T.I. $17,66 \%$, o que significa que tanto o pessoal da Emergência como o pessoal da U.T.I., apresentavam um grau moderado de exaustão emocional.

Tabela 5 - Subescala de exaustão emocional relacionada ao local de trabalho

\begin{tabular}{llcc}
\hline $\begin{array}{l}\text { Setor em que trabalha } \\
\text { no hospital }\end{array}$ & Escore & Frequência & \% \\
\hline \multirow{3}{*}{ Emergência } & Baixo & 1 & 5 \\
& Moderado & 17 & 90 \\
\hline & Alto & 1 & 5 \\
\hline & Total & 19 & 100 \\
\hline \multirow{2}{*}{ U.T.I } & & 1 & 5 \\
& Baixo & 19 & 90 \\
\hline & Moderado & 1 & 5 \\
\hline
\end{tabular}

Fonte: Hospital X, Campina Grande-PB, 2009. 
Quanto aos valores correspondentes, a sub-escala de despersonalização em relação ao local de trabalho dos entrevistados revela que, aqueles que atuavam na emergência apresentaram frequência de escore e porcentagem respectivamente: baixo de $10-$ $53 \%$, moderado de $6-31 \%$ e alto de $3-16 \%$. Os que atuavam em UTI., apresentaram frequência de escore e porcentagem de: baixo de $7-33 \%$, moderado de $9-43 \%$, e alto de 5 - 24\%. A média extraída correspondente à emergência é de $7,89 \%$, enquanto que a média da U.T.I., é igual a 9,28\%. Diante destes valores, podemos concluir que ambos apresentam um índice moderado de despersonalização, mas que os profissionais da U.T.I., apresentam uma média discretamente mais elevada se comparada à média da emergência.

Embora o termo despersonalização possa remeter a uma situação em que o profissional deixou de ter sua personalidade, também reflete uma situação em que o EU do indivíduo passou e vem passando por transformações, levando-o a agir de maneira fria e impessoal com seus colegas e com os receptores de seu cuidado, gerando atitudes de cinismo e ironia e demonstrando pouco interesse sobre o que pode vir a acontecer com as pessoas em sua volta (BENEVIDESPEREIRA, 2002).

No que se diz respeito aos escores da sub-escala de sentimento de incompetência profissional relacionados ao local de trabalho dos sujeitos entrevistados, aqueles que atuavam em emergência, revelaram frequência de escore moderado de 16, o que corresponde a $84 \%$, e alto de 3 , com uma porcentagem de $16 \%$; já aqueles que atuavam em UTI, só foi identificado escore moderado de 21 , correspondendo a 100 por cento. A média encontrada foi de 18 , para a equipe da emergência, e de $16,52 \%$ para a equipe da U.T.I., o que possibilita afirmar que as duas equipes apresentam um nível moderado de sentimento de incompetência profissional.

$\mathrm{O}$ resultados correspondente aos valores da sub-escala de exaustão emocional entre os que trabalham em outra instituição, além da instituição fonte da pesquisa, e os que não trabalham, pode-se inferir que os escores moderados entre aqueles atuantes em outra instituição, revelaram um valor moderado de 25, com uma porcentagem de $93 \%$, e com frequência de escore alto de 2 , com $7 \%$ de porcentagem. Para aqueles que não atuavam em outra instituição, o escore baixo foi 2, com porcentagem de $15 \%$ e, moderado de 11 e $85 \%$. A média encontrada para os que trabalhavam em outra instituição corresponde a 18 e a média dos que não trabalhavam corresponde a 16,76, o que denota que ambos se encontram em um nível moderado de exaustão emocional, mas que de acordo com a média, há uma pequena elevação para os profissionais que exercem atividade extra-hospitalar se, comparado aos que não exercem.

A procura por um segundo emprego na área da enfermagem se dá por conta da 
necessidade de buscar uma renda condizente para o sustento da família, uma vez que devido à situação econômica e aos baixos salários pagos a esses profissionais, fica praticamente impossível receber uma remuneração suficiente, frente às despesas mínimas com apenas um emprego. Portanto, o profissional se submete a uma dupla jornada, o que vai interferir diretamente em sua qualidade de vida (PAFARO; MARTINO, 2004).

Os escores da sub-escala de despersonalização em relação aos profissionais que exercem atividade em outra instituição e os que não exercem, revelaram que para aqueles que desenvolviam atividades em outra instituição, o escore baixo estava com uma frequência de 11 e porcentagem de $41 \%$, escore médio de 9 e porcentagem de $33 \%$ e, escore alto de 7 e porcentagem de 26\%. Porém, para aqueles não atuantes em outras instituições, apresentaram tanto escore baixo, quanto moderado de 6 , o que corresponde a uma porcentagem de $46 \%$ e apenas um com níveis de escore alto. Com isso, percebe-se que média para os que trabalhavam é igual a 9,22, e para os que não trabalhavam é de 7,38, o que permite afirmar que ambos encontram-se em um grau moderado de despersonalização.

A baixa remuneração tem sido apontada como sendo uma variável de extrema importância. Inclusive é também reconhecida como sendo um dos pontos de desequilíbrio entre empresa e trabalhador. A baixa remuneração faz com que os profissionais de enfermagem se submetam à dupla ou até mesmo tripla jornada de trabalho em busca de melhorar sua situação financeira, o que acaba deixando-os esgotados e sem muita energia para despender durante a execução de suas atividades (MASLACH; LEITER, 1999).

Quanto aos resultados da sub-escala de sentimento de incompetência profissional relacionada aos profissionais que trabalham em outra instituição e os que não trabalham, revelou que, os que trabalhavam em outra instituição apresentaram escore baixo com frequência de 1 e porcentagem de 4\%; escore moderado com frequência de 26 e porcentagem de 96\%; e aqueles que não atuavam em outra instituição apresentou escore moderado de 10 , o que correspondeu a $77 \%$ e, um escore alto de 3,correspondendo a 23\%. Diante dos valores medianos expostos, podemos concluir que ambos encontram-se em um grau moderado de sentimento de incompetência profissional, porém pelo fato de aqueles que não trabalhavam em outra instituição apresentarem uma maior média, indica que os mesmos sentem-se mais satisfeitos e motivados com seu trabalho.

Benevides-Pereira (2002) orienta que existe uma maior propensão ao Burnout naquelas pessoas que possuem uma maior sobrecarga de trabalho, pois ocupa boa parte do seu tempo, inclusive aquele que poderia ser dedicado ao descanso, ao lazer e até mesmo à busca pela atualização profissional, 
o que vai gerar nas pessoas uma insatisfação maior e uma certa insegurança nas atividades a serem desempenhadas.

Todos se encontram em grau moderado quando se fala em exaustão emocional, mas os valores mais elevados foram para os que trabalhavam no turno diurno, seguido pelos que trabalhavam nos dois turnos e por fim os menores valores foram para os que trabalhavam no período noturno.

Sabe-se que o trabalho noturno é mais desgastante, sendo ele também o maior responsável por perturbações físicas e psíquicas que ocorrem nos profissionais que se submetem a este turno de trabalho, pois o organismo está acostumado ao trabalho diurno e ao descanso noturno. Assim sendo, o trabalho noturno exige que o corpo trabalhe em um período que ele está acostumado a descansar (MARTINS, 2002).

Não há aceitação quanto à insatisfação no trabalho ser a causa ou efeito do desenvolvimento da síndrome de Burnout. Porém, o que se pode ser afirmado é que existe uma correlação inversa entre a síndrome do esgotamento profissional e satisfação nas atividades ocupacionais desempenhadas (BENEVIDES-PEREIRA, 2002).

Quando as pessoas se sentem ineficientes, provam constantemente de um sentimento de inadequação, perdendo a capacidade de acreditar que podem fazer a diferença perante os outros e desacreditando em seu próprio potencial, o que vai levar as outras pessoas a também duvidarem e até mesmo perderem a confiança que antes tinham nela (MARTINS, 2002).

É de conhecimento de todos que os serviços de saúde, em especial os hospitais, oferecem um ambiente insalubre e de condições de trabalho frequentemente precárias para seus funcionários, sendo possivelmente a pior dentre as demais verificadas em outros setores de atividade. Além de oferecer riscos de acidentes para os profissionais e de doenças propriamente ditas, ainda tem a atividade desenvolvida pelos profissionais de enfermagem que vem a contribuir decisivamente para o surgimento de doenças relacionadas ao ambiente de trabalho (BARROS, et al, 2008; MARTINS, 2002; MASLACH; LEITER, 1999; PAFARO; MARTINO, 2004; SILVA; MARZIALE, 2002).

As causas do adoecimento da equipe de enfermagem têm estreitas relações com as inadequadas condições de trabalho aliadas com o acúmulo de vínculos de trabalho, com consequente sobrecarga de trabalho associados à submissão diária a condições desfavoráveis no ambiente de trabalho, contribuindo assim sobremaneira para a redução na qualidade dos serviços prestados (SILVA; MARZIALE, 2002).

\section{Considerações Finais}

Hoje não há mais dúvidas que o ambiente de trabalho pode sim ser fator de risco para o desenvolvimento de patologias 
ocupacionais, uma vez que o local de trabalho não oferecendo condições adequadas, tanto em questão de recursos materiais e infraestrutura, quanto os recursos humanos necessários para o desempenho de cada função, pode ocasionar em seus funcionários um desgaste e um sofrimento físico e/ou mental.

O comparativo realizado entre os turnos de trabalho e as sub-escalas da síndrome denota que para a subescala de exaustão emocional a diferença é sutil e, independentemente do turno de trabalho, o escore é moderado. Já para a subescala de despersonalização os profissionais que trabalhavam no período diurno e os que trabalham no período noturno apresentam um escore moderado, enquanto os profissionais que trabalham os dois períodos, diurno e noturno, apresentam um escore baixo. $\mathrm{Na}$ escala de sentimento de incompetência profissional o escore encontrado foi moderado para todos os turnos.

Foi realizado também um comparativo entre os profissionais que exercem atividade em outra instituição e os que não trabalham em relação às subescalas da síndrome. Os valores encontrados indicam uma diferença pouco significativa entre eles e ambos apresentam um escore moderado para as três dimensões da síndrome.

Os resultados encontrados permitem afirmar que está havendo pontos de desequilíbrio entre os profissionais e a instituição e essa situação está levando o profissional ao desgaste. O nível moderado já serve de alerta para a observância da presença ou não dos sinais e sintomas referentes à síndrome e possíveis intervenções que podem ser realizadas, tais como o levantamento do quadro de pessoal para saber se realmente condiz com a necessidade do hospital, ou está havendo sobrecarga de trabalho, a fim de minimizar os danos do esgotamento e tencionar o compromisso com o trabalho tornando-o mais eficiente e eficaz.

\section{Referencias Bibliográficas}

BARROS, D. S. et al. Médicos plantonistas de unidades de terapia intensiva: perfil sóciodemográfico, condições de trabalho e fatores associados à síndrome de Burnout. Rev. bras. ter. intensiva. v. 20, n. 3, p. 235-240, 2008.

BENEVIDES-PEREIRA, A. M. T. As atividades de enfermagem em um hospital: um fator de vulnerabilidade ao Burnout. In: Benevides-Pereira AMT. (Org). Burnout: quando o trabalho ameaça o Bem-estar do trabalhador. São Paulo: Casa do Psicólogo, 2002.

CARLOTTO, M. S.; CÂMARA, S.G. Análise da produção científica sobre a síndrome de Burnout no Brasil. Psico. v. 9, n. 2, p. 152158, 2008.

LAUTERT, L. O desgaste profissional do Enfermeiro. 1995. 300 f. Tese (Doutorado) Curso de Psicologia, Departamento de 
Faculdade de Psicologia, Universidad Pontifícia Salamanca, Espanha, 1995.

MARTINS, M. M. Qualidade de vida e capacidade para o trabalho dos profissionais em enfermagem no trabalho em turnos. 2002. 85 f. Dissertação (Pós-graduação) - Curso de Engenharia de Produção, Universidade Federal de Santa Catarina, Florianópolis.

MASLACH, C.; LEITER, M. Trabalho: fonte de prazer ou desgaste?. Campinas: Papirus, 1999.

MASLACH, C.; JACKSON, S. The measurement of experience. Burnout Occup Behav. v. 2, p. 99-113, 1981.

MULLER, D. V. K. A Síndrome de Burnout no trabalho de assistência a saúde: Estudo junto aos profissionais da equipe de enfermagem do Hospital Santa Casa de Misericórdia de Porto Alegre. 2004. 110 f. Dissertação (Mestre) - Curso de Engenharia, Departamento de Psicologia Social e Institucional, Universidade Federal do Rio Grande do Sul, Porto Alegre, 2004.

MUROFUSE, N. T.; ABRANCHES, S. S.; NAPOLEÃO, A. M. A. . Reflexões sobre o estresse e Burnout e a relação com a enfermagem. Rev. latino-am. Enferm. v. 13, n. 2, p. 255-261, 2005.
REISDORFER, M. C. T. Condicionantes organizacionais relacionadas à atuação do enfermeiro no trabalho: uma abordagem ergonômica. 2002. 148 f. Dissertação (Pósgraduação) - Departamento de Engenharia de Produção e Sistemas, Universidade Federal de Santa Catarina, Florianópolis.

PAFARO, R. C.; MARTINO, M. M. F. Estudo do estresse do enfermeiro com dupla jornada de trabalho em um hospital de oncologia pediátrica de Campinas. Rev. esc. de enferm. USP, Campinas, v. 2, n. 38, p. 152-160, 2004.

ROCHA, P. K. et al. Cuidado e tecnologia: aproximações através do modelo de cuidado.

Rev. Bras. Enferm. v. 61, n. 1, p. 113-116, 2008.

ROSA, C.; CARLOTTO, M.S. Síndrome de Burnout e satisfação no trabalho em profissionais de uma instituição hospitalar. Rev. soc. bras. Pesq. Histórica. v. 8, n. 2, p. $1-15,2005$.

SILVA, D. M. P. P.; MARZIALE, M. H. P. O adoecimento da equipe de enfermagem e o absenteísmo doença. Rev. ciênc. cuidado. saúde, Maringá, v. 1, n. 1, p. 133-136, 2002.

STACCIARINI, J. M. R.; TRÓCCOLL, B. T. $\mathrm{O}$ estresse na atividade ocupacional do enfermeiro. Rev. latino-am. Enferm. v. 9, n. 2, p. 17-25, 2001. 